\title{
Comparison Among Phonation of the Sustained Vowel $/ \varepsilon /$, Lip Trills, and Tongue Trills: The Amplitude of Vocal Fold Vibration and the Closed Quotient
}

\author{
Gislaine Ferro Cordeiro, Arlindo Neto Montagnoli \\ and Domingos Hiroshi Tsuji \\ University of São Paulo School of Medicine \\ Brasil
}

\section{Introduction}

Trill exercises are traditionally used in the clinical practice of speech-language pathology as vocal warm-ups in the treatment of dysphonia (Behlau \& Pontes, 1995; Sataloff, 1991). They are also used by voice coaches (Aydos \& Hanayama, 2004; Scarpel \& Pinho, 2001) in the training of professional voice users, such as singers, actors (including voice actors), teachers, and lawyers. Although there are various types of trill exercises, including gargling, voiced fricatives, and simultaneous tongue/lip trills, the most commonly used exercises are tongue trills and lip trills (Scwarz \& Cielo, 2009; Menezes et al., 2005).

In the literature, the first reports of trill exercises date from the 1970s, at which time the tongue trill was already considered a "universal technique," i.e., a technique that can change the overall quality of the voice (Linklater, 1976). Trills are among the so-called facilitating sounds (Behlau \& Pontes, 1995), and trill exercises can be used in the treatment of hyperkinetic and hypokinetic disorders (Schneider \& Sataloff, 2007; Speyer, 2008); they can also be used as vocal warm-ups (Aydos \& Hanayama, 2004; Speyer, 2008). Trill exercises are therefore widely disseminated among voice coaches, including speech-language pathologists, singing teachers, and drama teachers (Aydos \& Hanayama, 2004). In individuals with a normal voice, tongue trills increase the amplitude of vocal fold vibration, reduce the glottal gap (Rodrigues, 2001), and improve the results of auditory-perceptual and acoustic analyses, resulting in less shimmer, a higher harmonics-to-noise ratio, increased amplitude of the harmonics, and decreased noise (Schwarz \& Cielo, 2009; Rodrigues, 1995). During tongue trills, the entire laryngeal framework vibrates and there is anteroposterior constriction of the pharynx (Bueno, 2006). After the performance of tongue trills, the fundamental frequency increases, broad- and narrow-band spectrograms become clearer, and glottic closure increases, as do the amplitude and symmetry of vocal fold vibration, all of which demonstrate that tongue trills change the glottal source and vocal tract filter (Scwarz \& Cielo, 2009).

Speech-language pathologists also use tongue trills, principally in individuals diagnosed with vocal nodules (Bueno, 2006). In addition, tongue trills can be used in individuals with 
chronic edema and in those with hyperfunctional dysphonia (Pinho \& Pontes, 2008). Some authors also recommend tongue trills in cases of hypofunctional dysphonia (Behlau e Pontes, 1995). Manieka-Aleksandrovix (2006) collected data regarding 500 patients with aphonia due to psychogenic dysphonia and found that gargling is one of the exercises used on the first day of therapy for voice rehabilitation.

Casper et al. (1992) employed tongue trills as a therapeutic resource in individuals with vocal fold paralysis and in those having undergone laryngeal surgery (Woo et al., 1994). The tongue trill is contraindicated for individuals with recent-onset acute inflammation, because the exercise can aggravate the inflammatory phase. In the immediate postoperative period, tongue trills can delay healing (Pinho \& Pontes, 2008) and, in cases of papillomatosis, stimulate the dissemination of the disease (Pinho \& Tsuji, 2006).

When performing trill exercises, individuals should keep the tongue (or lips) and the mandible relaxed, coordinating the airflow so that vibration can occur (Scneider \& Sataloff, 2007). The tongue trill is maintained by the interaction among the firmness of the body of tongue, control of the tip of the tongue, glottic closure, and control of the exhaled air. The exercise should be performed with the sides of the body of tongue firmly pressed against the dental alveoli and the tip of the tongue positioned in the region of the incisive papilla, free to vibrate (McGowan, 1992). As a result, the entire vocal tract vibrates (Scwarz \& Cielo, 2009). For lip vibration (lip trill) to occur, the lips should be held together tightly enough to promote airway occlusion and relaxed enough for air pressure to overcome the resistance (Gaskill e Erickson, 2008). During lip trills, as during tongue trills, there is interaction among the vocal tract, glottal vibration, and the exhaled air during lip trills (Titze, 2006). During all trill exercises, the vibrating organ acts as a valve and creates oscillatory differences in external pressure and in the pressure in the cavity behind the constriction. This produces differences in the pressure, speed, and volume of air in the oral cavity, causing changes in the pharyngeal wall. Therefore, for vocal fold vibration to occur concomitantly with the point of oscillation of the oral cavity, subglottic air pressure must be greater than is that during normal phonation (McGowan, 1992).

Tongue and lip vibration follow the same principle as does the vocal fold mass effect: the anterior part of the vocal tract is occluded by the tip of the tongue or the lips. Intraoral pressure becomes greater than the atmospheric pressure and therefore greater than the force that maintains the anterior part of the vocal tract closed. Therefore, the anterior part of the vocal tract opens and is subsequently "sucked out" by the speed of the airflow (McGowan, 1992).

According to Gaskill and Erickison (2008), the difference between lip trills and other exercises that focus on the anterior part of the vocal tract is that the lip trill is the only exercise that promotes lip occlusion and non-occlusion (without loss of muscle tone). This causes the lips to vibrate, although at a frequency lower than that of vocal fold vibration. Therefore, airflow and subglottic pressure must adjust in order to allow the lips and the vocal folds to vibrate, overloading the vocal folds. The variations that occur in the pharynx during lip trills can increase the force of mucosal vibration during the wavelike motion of the vocal folds (McGowan, 1992).

Because of high vocal demand, professional voice users should maintain the fitness of all of the structures involved in phonation. For professional voice users, voice training should 
address a wide range of issues, from breathing to voice articulation and projection. Trill exercises involve a functional balance among the vibrating organ (the tongue, the lips, or a combination of the two), the vocal tract, the larynx, and the exhaled air. Therefore, they constitute one of the principal tools for vocal warm-up and training in professional voice users (Aydos \& Hanayama, 2004; Nix, 1999).

Studies involving electroglottography (EGG) and comparing the closed quotient during lip trills with that during phonation of the vowel /a/ before and after the exercise showed a reduction of approximately $50 \%$ in the closed quotient during the performance of lip trills (Gaskill \& Erickson, 2008) and an increase in the value when the exercise was performed at high intensity by singers (Cordeiro et al., in prelo). The change is more evident in untrained individuals. The mechanical interaction between the source and the filter plus a lower adduction of the vocal process were reported to be responsible for the results obtained(15). The closed quotient varies widely during trill exercises (Cordeiro et al., in prelo).

Recent studies have attempted to determine the best exercise prescription (the most effective duration and number of repetitions per session) for each case. Menezes et al.. (2005) suggested that, for individuals with no complaints or dysphonia, trill exercises be performed for a maximum of three and five minutes by females and males, respectively. For females with nodules, the ideal exercise duration is five minutes, the exercise leading to signs of vocal fatigue if performed for seven minutes or more (Menezes, 2011). , According to Schwarz (2009), when prescribing the exercise, the vocal resistance of the individual should be taken into consideration. In the study conducted by Schwarz (2009), the individuals performed three series of fifteen trills with a 30-second interval of passive rest between each series, and the author reported that voice quality improved after the exercise.

In this chapter, we determine whether there are differences among tongue trills, lip trills, and phonation of the sustained vowel $/ \varepsilon /$ in classically trained singers in terms of the maximum amplitude of vocal fold vibration, mean closed quotient, and standard deviation of the closed quotient, as assessed by EGG. We chose to compare the vocal fold vibration seen during lip and tongue trills with that seen during phonation of the sustained vowel $/ \varepsilon$ / because that vowel is considered an open vowel, produced with minimal constriction of the vocal tract (Story, 1998; Gregio, 2006).

\section{Study sample and method}

\subsection{Ethical aspects}

The present study was approved by the Research Ethics Committee of the Hospital das Clínicas da Faculdade de Medicina da Universidade de São Paulo (HCFMUSP, University of São Paulo School of Medicine Hospital das Clínicas; Protocol no. 907/06, February 14, 2007; Appendix 1), located in the city of São Paulo, Brazil. All participating individuals gave written informed consent.

\subsection{Study sample}

In the present study, we evaluated 14 individuals ( 7 males and 7 females). We applied the following criteria: 
- inclusion criteria - being a healthy, classically trained, professional singer; having laryngeal control; having mastered the techniques for performing lip and tongue trills; and presenting with no vocal fold lesions

- exclusion criteria-having been a professional singer for less than three years; presenting with singing or speaking voice complaints; presenting with incomplete glottal closure; having reported intolerance to examination of the larynx

A total of 4 individuals were excluded from the present study. Of those, 2 were male and 2 were female. Of the 2 males, 1 was excluded because he could not tolerate the examination of the larynx and 1 was excluded because he had been a professional singer for less than three years (one year and six months). Of the 2 females, 1 was excluded because she could not tolerate the examination of the larynx and 1 was excluded because she presented with speaking voice complaints.

A total of 10 singers were analyzed. Their characteristics are shown in Table 1.

\begin{tabular}{ccccl}
\hline Individual & Gender & Age & $\begin{array}{c}\text { Years singing } \\
\text { professionally }\end{array}$ & Voice type \\
\hline 1 & Female & 24 & 4 & Soprano \\
2 & Female & 45 & 20 & Soprano \\
3 & Female & 30 & 3 & Mezzo-soprano \\
4 & Female & 30 & 4 & Mezzo-soprano \\
5 & Female & 48 & 15 & Contralto \\
6 & Male & 29 & 5 & Tenor \\
7 & Male & 27 & 6 & Tenor \\
8 & Male & 33 & 12 & Baritone \\
9 & Male & 34 & 15 & Baritone \\
10 & Male & 38 & 18 & Bass \\
\hline
\end{tabular}

Table 1. Characteristics of the individuals included in the study sample

\subsection{Method}

\subsubsection{Preparation for data collection}

Before data collection, we measured the vocal range of each participant. Vocal range is the distance between the lowest and the highest note that an individual can produce, excluding the vocal fry register and including the falsetto.

After having measured the vocal range of the participants, we selected the 5th whole step above the lowest possible note that each individual was able to produce (Cooper, 1979). The participants were then asked to produce, in that note and with their larynx in a low position, the sustained vowel $/ \varepsilon /$, lip trills, and tongue trills, as well as the voiced fricatives $/ v /, / z /$, 
and $/ 3 /$, at the highest and lowest possible intensities of which they were capable. As shown in Table 2, we chose the lowest of the highest intensities and the highest of the lowest intensities, in order to standardize the intensities during exercise training and data collection. The voiced fricatives were used because the lowest of the highest intensities is commonly achieved during the phonation of those sounds; therefore, the participants were able to perform the tests comfortably without experiencing vocal fatigue or aperiodicity due to the use of threshold-range phonation (Jiang et al., 2001) during the performance of trill exercises.

\begin{tabular}{lcccccc}
\hline Intensity & Vowel $/ \varepsilon /$ & Lip trill & Tongue trill & $/ \mathrm{v} /$ & $/ \mathrm{z} /$ & $/ 3 /$ \\
\hline Highest $(\mathrm{dB})$ & 51 & 52 & 52 & $<50$ & $<50$ & 51 \\
Lowest $(\mathrm{dB})$ & 85 & 70 & 71 & 68 & 70 & 71 \\
\hline
\end{tabular}

Table 2. Highest and lowest intensities achieved by individual 1 during sustained phonations. The values chosen for data collection were $52 \mathrm{~dB}$ for the lowest intensity and 68 $\mathrm{dB}$ for the highest intensity

In order to measure vocal range, as well as to determine and maintain the selected note, we used a Casio VL-Tone-VL1 keyboard (Casio Computer Co., Ltd., Tokyo, Japan). Intensity was measured with a sound pressure level meter (model 33-2055, RadioShack Corporation, Fort Worth, TX, USA) placed $30 \mathrm{~cm}$ from the corner of the mouth of the singer.

After the abovementioned procedure, each singer practiced producing the sounds at the requested note and at the intensity selected (maximum variation, $2 \mathrm{~dB}$ ), with minimal effort and maintaining the larynx in the same (low) position, for $\geq 10$ seconds. The practice session was conducted with the aid of a speech-language pathologist specializing in voice, who instructed the singers during a meeting held before data collection. On the day of data collection, previously agreed upon by the singers and the investigators, the phonation tasks were performed again before each test.

Participants were informed of the objectives and conditions of the present study before the first evaluation was scheduled. If a participant presented with illness, sleep deprivation, vocal fatigue, or dysphonia on the day of data collection, the evaluation was rescheduled.

\subsubsection{Data collection and analysis}

The individuals underwent videolaryngostroboscopy by an experienced otolaryngologist of the HCFMUSP Clinical Otolaryngology Division Voice Group Outpatient Clinic and EGG at the Centro de Especialização em Fonoaudiologia Clínica (CEFAC, Center for Clinical Audiology and Speech-Language Pathology), both located in the city of São Paulo. The tests were performed on different days so that the singers did not experience vocal fatigue. For the tests, the individuals were asked to produce the sustained vowel $/ \varepsilon /$ and perform tongue and lip trills for as long as possible at the same frequency and intensities as those used in the training sessions (5th whole step above the lowest note in their range and the lowest of the highest/highest of the lowest, respectively). Frequency was controlled by the singers. In order to do that, they used the same keyboard that was used in the training sessions. Intensity was monitored by a speech-language pathologist in the examination room, using 
the same sound pressure level meter that was used in the training sessions, which was again positioned at $30 \mathrm{~cm}$ from the corner of the mouth of the singer. We made intraindividual comparisons. Low-intensity phonations were compared only with one another, as were high-intensity phonations.

\subsubsection{Videolaryngostroboscopy}

We used videolaryngostroboscopy in order to measure the amplitude of vocal fold vibration during the exercises. The singers were instructed to sit in a chair, with both hips well supported, head held straight, and the chin at a $90^{\circ}$ angle to the neck. In order to maintain the head of each participant fixed in position, the chair was equipped with a headrest that was adjusted to the height of the individual and a piece of foam that was covered with comfortable fabric, measuring $14 \mathrm{~cm}$ in width, $10 \mathrm{~cm}$ in height, and $7 \mathrm{~cm}$ in depth. In addition, their heads were secured to the headrest with a headband (Figure 1).

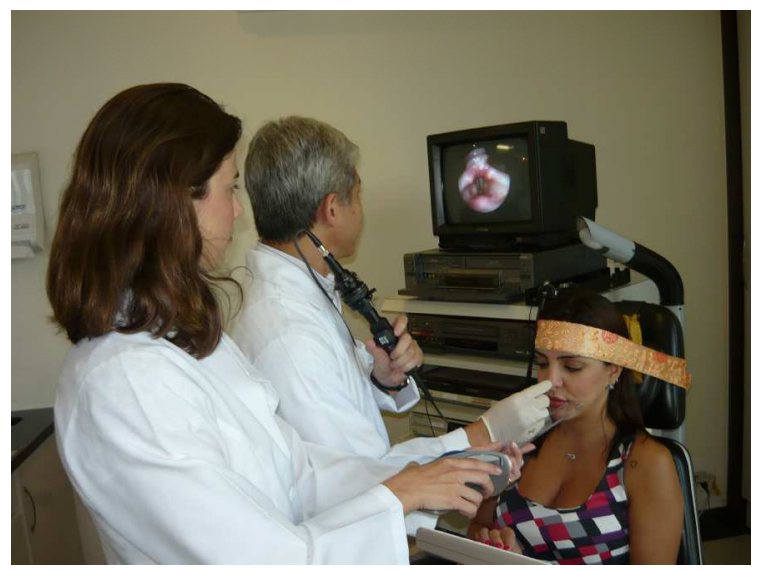

Fig. 1. Photograph of a singer undergoing videolaryngostroboscopy.

Images of vocal fold vibration were obtained with a stroboscopic light source (4914; Brüel \& Kjær Sound \& Vibration Measurement A/S, Nærum, Denmark) and captured with a 3.2$\mathrm{mm}$ fiberoptic laryngoscope (ENT-30PIII; Machida Endoscope Co., Ltd., Tokyo, Japan), positioned so that the region of the arytenoid cartilages and the vocal folds were fully visible. In addition, we attempted to keep variations in the angle and distance of recording to a minimum. To that end, the otolaryngologist held the fiberoptic laryngoscope between the thumb and middle finger, with the index finger resting on the tip of the nose of the individual being examined (Figure 1).

The fiberoptic laryngoscope was connected to a charge-coupled device camera (IK-M41A; Toshiba, Tokyo, Japan), and the images were recorded on videotape with an NTSC videocassette recorder (NV-FS90; Panasonic Corporation, Osaka, Japan). The images were digitized on a computer (Inspiron 1525; Dell, Inc., Round Rock, TX, USA) equipped with a 1.73-GHz Pentium Duo CPU T2370 processor (Intel Corporation, Santa Clara, CA, USA), 2 GB of RAM, and a 32-bit operating system. In order to digitize the images, we used a video capture card (PCTV Pro USB; Avid Technology, Inc., Burlington, MA, USA) and the program Studio QuickStart, version 10.8 (Pinnacle/Avid Technology, Inc., Burlington, MA, 
USA). The digitized images were analyzed frame by frame, and, for each individual, we obtained photographs of the following phonations at their peak amplitudes:

- low intensity-sustained vowel / $/$ /, 5 photographs; lip trills, 3 photographs; and tongue trills, 3 photographs

- high intensity-sustained vowel / $\varepsilon /, 5$ photographs; lip trills, 3 photographs; and tongue trills, 3 photographs

The photos were edited with Adobe Photoshop CS2 software, version 9.0 (Adobe Systems Incorporated, San Jose, CA, USA), which allowed better visualization of the amplitude of vocal fold vibration, as well as of the limits of the region of the right arytenoid cartilage (Figures 2a and 2b).

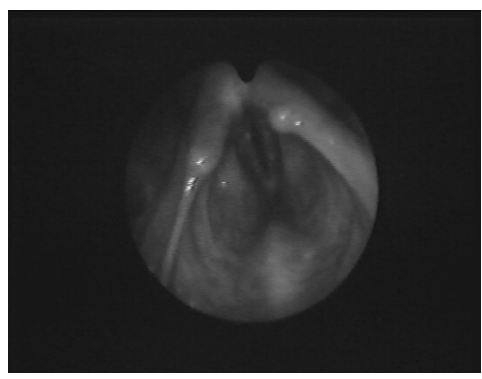

a

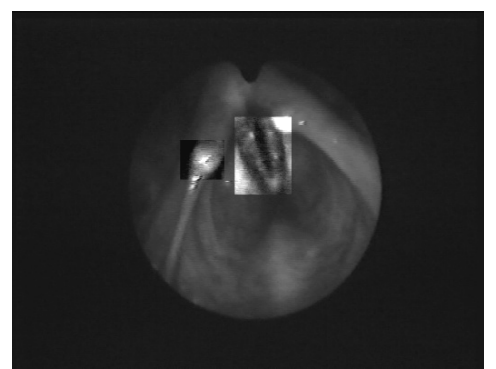

b

Fig. 2. Photograph of the maximum amplitude of vocal fold vibration during lip trills. In a, unedited image; and in $b$, edited image.

In order to measure the maximum amplitude of vibration, we imported the edited images into the program X-Cade, version 2.0, specifically designed for the present study by Dr. Arlindo Neto Montagnoli, an engineer at the Federal University of São Carlos School of Electrical Engineering, located in the city of São Carlos, Brazil. Measurements were taken from a total of 220 photographs.

In order to reduce the number of measurement errors caused by variations in the position of the fiberoptic laryngoscope, we also measured the length of the anatomical structure near the arytenoid region. The structure is a cuneiform cartilage covered by the arytenoid mucosa and was used as a reference for comparison. That structure was chosen because its configuration is not changed by the phonation task being performed. We first measured the largest diameter of the abovementioned structure, which the software automatically converted into a comparative reference that was used in order to measure the amplitude of vocal fold vibration. Therefore, when we refer to the amplitude of vocal fold vibration as being 0.5 , this means that the amplitude value corresponds to half the measurement of the region corresponding to the cuneiform cartilage (Figure 3 ).

All of the measurements were taken in a blinded fashion by the same rater, who, for each individual evaluated, adopted the following self-calibration method: before considering a measurement to be valid, the rater measured the images obtained from a given individual 
several times until the measurements taken from the same image were within two decimal places of each other. The images from each individual were measured without interruption. Otherwise, the calibration process was restarted, and the measurements were taken again. The data were tabulated and entered into a database for subsequent statistical analysis.

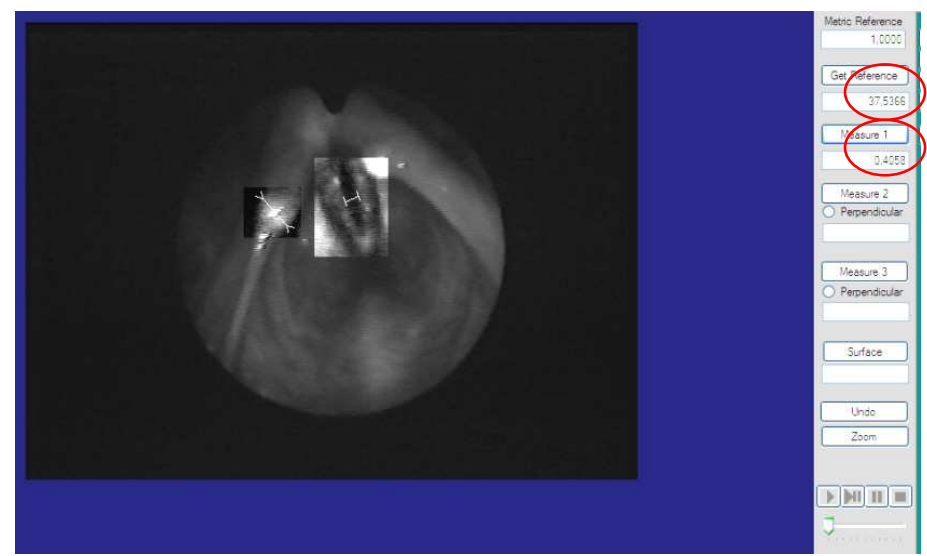

Fig. 3. X-Cade software. The amplitude measurement corresponds to 0.4 of the measurement of the arytenoid cartilage.

\subsubsection{EGG}

In order to measure the EGG signal, we referred the individuals to a sound-treated booth in a quiet room in the CEFAC Voice and Speech Laboratory. We used an EG2-Standard electroglottograph (Glottal Enterprises, Syracuse, NY, USA). The electroglottograph was connected to a computer audio interface (BCA2000; MUSIC Group Services EU GmbH, Willich, Germany), which was in turn connected to a computer equipped with a 1.66-GB Centron processor (Advanced Micro Devices, Inc., Sunnyvale, CA, USA).

The individuals were asked to remove any metal objects that they might be wearing in the head and neck region and to sit upright in a chair equipped with a headrest. To ensure the safety of the individuals, we placed a rubber mat under the chair. The neck region was cleaned with dry paper towel. We applied a thin layer of hypoallergenic electrically conductive gel (SPECTRA 360 ${ }^{\circledR}$; Parker Laboratories, Inc., Fairfield, NJ, USA) to the electrodes, which were placed over each ala of the thyroid cartilage and secured with a Velcro strap around the neck. To ensure that the electrodes were positioned correctly, we asked the participants to produce the sustained vowel and perform tongue trills. We then observed whether the green LEDs of the top LED array (Electrode Placement/Laryngeal Movement) on the front panel of the electroglottograph were on. To confirm that there was signal, we observed whether the green LEDs of the bottom LED array (Signal) were on. To record the signal, we selected the Vocal Fold Contact Area signal option, and high or low gain was determined by monitoring the LED signal indicator. The signal was saved as a .wav file and edited with the audio editing suite Sound Forge, version 7.0, (Sony Creative Software, Inc., Middleton, WI, USA) at a sampling frequency of $22,050 \mathrm{~Hz}$ and a resolution of 16 bits. 
The EGG waveforms were submitted to high band-pass filtering with the program delay0.bat, designed by Maurílio Nunes Vieira, an engineer at the Federal University of Minas Gerais, located in the city of Belo Horizonte, Brazil. The waveforms were initially graded by two speech-language pathologists with experience in EGG and one engineer, in accordance with the grading system proposed by Vieira (1997):

- $\quad$ grade 1 , free from gross errors

- $\quad$ grade 2 , as accurate as possible

- $\quad$ grade 3, able to trace signals with irregular excitation

- $\quad$ grade 4, able to locate every individual glottal stop

Only grade 1 and grade 2 waveforms were included in the study. In order to take automatic measurements, we used the technique developed by Vieira (1997). We assessed the mean and standard deviation of the closed quotient for each task.

The closed quotient is the ratio between the closed phase and the complete cycle of the EGG waveform (Figure 4). The mean closed quotient is the measurement of each EGG waveform divided by the number of waveforms analyzed by the software. The standard deviation is the variation in that measurement over time.

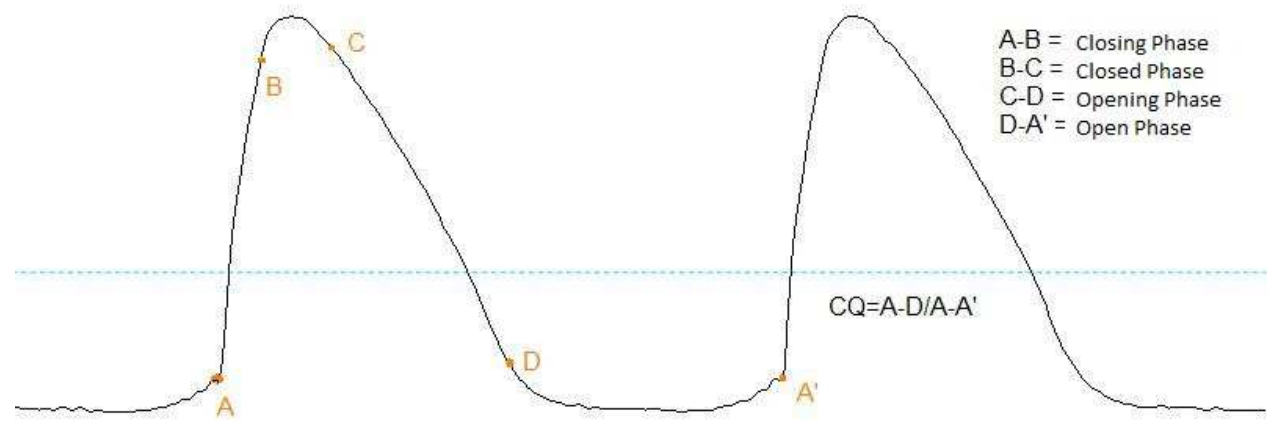

Fig. 4. Phases of the EGG waveform and the closed quotient (CQ).

\subsection{Statistical analysis}

In order to analyze the results, we adopted a level of significance of $5 \%(p=0.05)$ for all statistical tests. The Statistical Package for the Social Sciences, version 13.0 (SPSS Inc., Chicago, IL, USA) was used for the analysis. In order to determine the differences among phonation of the sustained vowel $/ \varepsilon /$, lip trills, and tongue trills, we used Friedman's test. 
For the cases in which the difference was statistically significant, we used the Wilcoxon signed rank test in order to identify the types that differed.

For the presentation of the results, the measurements taken automatically by the program were considered to constitute the intraindividual means and standard deviations, whereas the values obtained by statistical analysis of those results were considered to constitute the interindividual means and standard deviations.

\section{Results}

\subsection{Videolaryngostroboscopy}

During videolaryngostroboscopy, it was occasionally difficult to synchronize the stroboscopic illumination with the vocal fold vibration during lip and tongue trills. Therefore, during those tasks, we photographed only those moments of the cycles at which the maximum opening was evident. During the phonation of the sustained vowel $/ \varepsilon /$ task, we encountered no such difficulty.

Although the otolaryngologist maintained the fiberoptic laryngoscope in position, laryngeal movement caused variations in the distance between the larynx and the laryngoscope, which in turn caused variations in the images of the larynx. Among the 220 images analyzed in the present study, the larynx was too far from the laryngoscope in $163(74 \%)$. Of the remaining 57 images (26\%), $11(5 \%)$ were images of sustained vowel phonation, $19(9 \%)$ were images of lip trills, and $27(12 \%)$ were images of tongue trills. The measurements of vocal fold vibration amplitude are presented in Tables 3 through 6 and illustrated in Figures 5 and 6.

\subsubsection{Low intensity}

\begin{tabular}{|c|c|c|c|c|c|c|c|}
\hline \multirow[b]{2}{*}{ Variable } & \multicolumn{7}{|c|}{ Vibration amplitude } \\
\hline & $\mathrm{N}$ & Mean & SD & Minimum & Maximum & Median & $\mathrm{p}$ \\
\hline$/ \varepsilon /$ & 10 & 0.11 & 0.03 & 0.06 & 0.16 & 0.11 & \\
\hline Lip trills & 10 & 0.17 & 0.06 & 0.10 & 0.32 & 0.16 & $0.002 *$ \\
\hline Tngue trills & 10 & 0.15 & 0.04 & 0.08 & 0.21 & 0.16 & \\
\hline
\end{tabular}

*Friedman's test, among all three variables

Table 3. Maximum amplitude of vocal fold vibration during phonation of the sustained vowel $/ \varepsilon /$ compared with that of vocal fold vibration during tongue and lip trills at low intensity 
Comparison Among Phonation of the Sustained Vowel /ع/, Lip Trills,

\begin{tabular}{ll}
\hline Pair & $\mathrm{p}^{*}$ \\
\hline Lip trills versus sustained vowel $/ \varepsilon /$ phonation & 0.007 \\
\hline Tongue trills versus sustained vowel $/ \varepsilon /$ phonation & 0.005 \\
\hline Tongue trills versus lip trills & 0.677 \\
\hline
\end{tabular}

*Wilcoxon signed rank test

Table 4. Pairwise comparisons among phonation of the sustained vowel $/ \varepsilon /$, lip trills, and tongue trills at low intensity in terms of the amplitude of vocal fold vibration

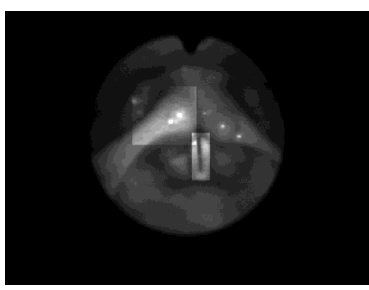

a

0.08

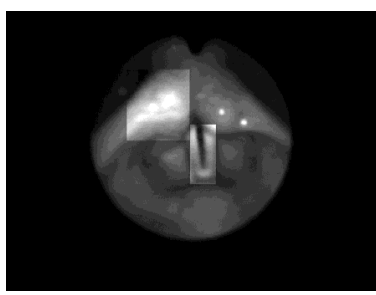

$\mathrm{b}$

0.11

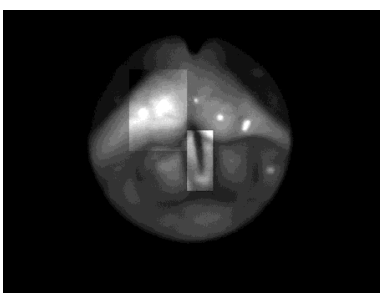

0.12

Fig. 5. Maximum amplitude of vocal fold vibration during phonation tasks at low intensity in relation to the cuneiform cartilage, as assessed by videolaryngostroboscopy. In a, phonation of the sustained vowel / $\varepsilon /$; in b, sustained lip trills; and in c, sustained tongue trills.

\subsubsection{High intensity}

\begin{tabular}{cccccccc}
\hline & & \multicolumn{7}{c}{ Vibration amplitude } & & \\
Variable & $\mathrm{N}$ & Mean & SD & Minimum & Maximum & Median & \\
\hline / $/$ L & 10 & 0.16 & 0.05 & 0.11 & 0.30 & 0.15 & \\
Lip trills & 10 & 0.29 & 0.08 & 0.16 & 0.40 & 0.30 & $0.001^{*}$ \\
Tongue trills & 10 & 0.26 & 0.12 & 0.15 & 0.50 & 0.21 & \\
\hline
\end{tabular}

*Friedman's test, among all three variables

Table 5. Maximum amplitude of vocal fold vibration during phonation of the sustained vowel $/ \varepsilon /$ compared with that of vocal fold vibration during tongue and lip trills at high intensity 


\begin{tabular}{ll}
\hline Pair & $\mathrm{p}^{*}$ \\
\hline Lip trills versus sustained vowel $/ \varepsilon /$ phonation & 0.005 \\
Tongue trills versus sustained vowel $/ \varepsilon /$ phonation & 0.005 \\
Tongue trills versus lip trills & 0.308 \\
\hline
\end{tabular}

*Wilcoxon signed rank test

Table 6. Pairwise comparisons among phonation of the sustained vowel / $\varepsilon /$, lip trills, and tongue trills at high intensity in terms of the amplitude of vocal fold vibration

The values obtained during phonation of the sustained vowel $/ \varepsilon /$ were significantly different from those obtained during lip and tongue trills at high and low intensities ( $\mathrm{p}>$ 0.01). The statistical tests revealed that the maximum amplitude of vocal fold vibration during phonation of the sustained vowel $/ \varepsilon /$ was significantly different from that of vocal fold vibration during lip and tongue trills. However, there were no significant differences between lip and tongue trills in terms of the maximum amplitude of vocal fold vibration.

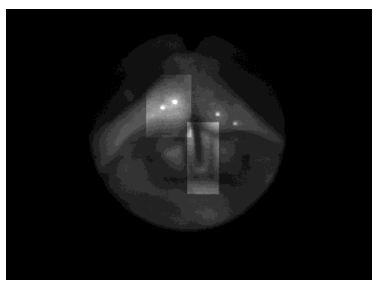

a

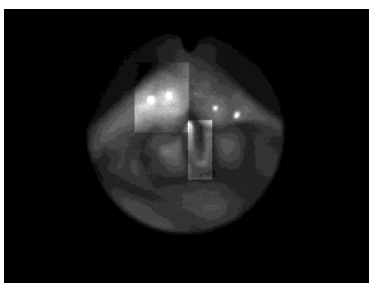

$\mathrm{b}$

0.19

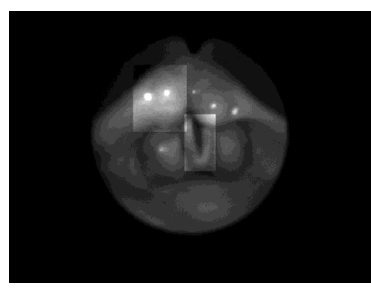

$\mathrm{C}$

0.22

Fig. 6. Maximum amplitude of vocal fold vibration during phonation tasks at high intensity in relation to the cuneiform cartilage, as assessed by videolaryngostroboscopy. In a, phonation of the sustained vowel $/ \varepsilon /$; in b, sustained tongue trills; and in c, lip trills.

\subsection{EGG - Closed quotient}

All of the EGG waveforms were either grade 1 or grade 2 in accordance with the criteria proposed by Vieira (1997) and were therefore appropriate for automatic measurements.

The measurements taken automatically by the program for analysis of the EGG waveform were considered to constitute the intraindividual means and standard deviations of the closed quotient. Those values were considered the study variables and placed on the vertical axes of the tables. The interindividual means and standard deviations of the closed quotient were obtained by statistical analysis and were placed on the horizontal axes of the tables.

Tables 7 through 10 show the results related to the intraindividual means and standard deviations of the closed quotient. 
3.2.1 Low intensity

\begin{tabular}{llccccccc}
\hline Measurement & Task & $\mathrm{N}$ & Mean & SD & Minimum & Maximum & Median & $\mathrm{p}^{*}$ \\
\hline $\begin{array}{l}\text { Intraindividual } / \varepsilon / \\
\text { mean of the lip trills }\end{array}$ & 10 & 47.72 & 12.27 & 31.87 & 67.27 & 51.64 & \\
$\begin{array}{l}\text { closed quotient } \\
\text { tongue trills }\end{array}$ & 10 & 50.97 & 12.96 & 33.93 & 71.48 & 53.36 & 0.301 \\
\multirow{2}{*}{$\begin{array}{l}\text { Intraindividual } / \varepsilon / \\
\text { SD of the lip trills }\end{array}$} & 10 & 3.12 & 3.40 & 0.88 & 12.18 & 1.99 & \\
closed quotient & 10 & 6.55 & 3.12 & 3.86 & 12.63 & 5.34 & 0.020 \\
\end{tabular}

*Friedman's test

Table 7. Closed quotient: number of samples, mean, standard deviation, minimum value, maximum value, median, and significance of the differences among phonation of the sustained vowel $/ \varepsilon /$, lip trills, and tongue trills at low intensity

\begin{tabular}{lll}
\hline Measurement & Pairs & $\mathrm{p}^{*}$ \\
\hline $\begin{array}{l}\text { Intraindividual SD } \\
\text { of the closed }\end{array}$ & Lip trills versus sustained vowel $/ \varepsilon /$ phonation & 0.059 \\
quotient & Tongue trills versus sustained vowel $/ \varepsilon /$ phonation & 0.037 \\
& Tongue trills versus lip trills & 0.508 \\
\hline
\end{tabular}

*Wilcoxon signed rank test

Table 8. Pairwise comparisons among phonation of the sustained vowel / $\varepsilon /$, lip trills, and tongue trills at low intensity in terms of the automatic measurements of significant values

\subsubsection{High intensity}

\begin{tabular}{|c|c|c|c|c|c|c|c|c|}
\hline Measurement & Task & $\mathrm{N}$ & Mean & SD & Minimum & Maximum & Median & $\mathrm{p}^{*}$ \\
\hline \multirow{3}{*}{$\begin{array}{l}\text { Intraindividual } \\
\text { mean of the } \\
\text { closed quotient }\end{array}$} & $/ \varepsilon /$ & 10 & 50.71 & 6.90 & 36.47 & 56.07 & 54.52 & \multirow{3}{*}{0.007} \\
\hline & lip trills & 10 & 59.21 & 12.57 & 42.85 & 82.73 & 54.68 & \\
\hline & tongue trills & 10 & 54.60 & 9.72 & 40.63 & 72.56 & 52.52 & \\
\hline \multirow{3}{*}{$\begin{array}{l}\text { Intraindividual } \\
\text { SD of the closed } \\
\text { quotient }\end{array}$} & $/ \varepsilon /$ & 10 & 1.62 & 0.84 & 0.77 & 3.17 & 1.39 & \multirow{3}{*}{0.020} \\
\hline & lip trills & 10 & 6.64 & 5.36 & 1.21 & 15.48 & 4.23 & \\
\hline & tongue trills & 10 & 4.75 & 2.78 & 1.68 & 9.50 & 4.15 & \\
\hline
\end{tabular}

*Friedman's test

Table 9. Automatic measurements, as taken by EGG: number of samples, mean, standard deviation, minimum value, maximum value, median, and significance of the differences among phonation of the sustained vowel $/ \varepsilon /$, lip trills, and tongue trills at high intensity 


\begin{tabular}{lll}
\hline Measurement & Pairs & $\mathrm{p}^{*}$ \\
\hline Intraindividual & Lip trills versus sustained vowel / $\varepsilon /$ phonation & 0.013 \\
mean of the & Tongue trills versus sustained vowel / $\varepsilon /$ phonation & 0.139 \\
closed quotient & Tongue trills versus lip trills & 0.017 \\
Intraindividual & Lip trills versus sustained vowel / $\varepsilon /$ phonation & 0.017 \\
SD of the closed & Tongue trills versus sustained vowel / $\varepsilon /$ phonation & 0.013 \\
quotient & Tongue trills versus lip trills & 0.114 \\
\hline
\end{tabular}

*Wilcoxon signed rank test

Table 10. Pairwise comparisons among phonation of the sustained vowel / $\varepsilon /$, lip trills, and tongue trills at high intensity in terms of the automatic measurements of significant values

\section{Discussion}

Our results show that, in general, tongue trills and lip trills were similar in terms of the amplitude of vocal fold vibration. However, there were differences between tongue trills and vowel $/ \varepsilon /$ phonation, as well as between lip trills and vowel $/ \varepsilon /$ phonation, in terms of the amplitude of vocal fold vibration.

We measured the largest diameter of the region corresponding to the cuneiform cartilage as a reference to the maximum amplitude of vocal fold vibration in order to make the measurements more reliable. The individuals were their own controls, and the amplitude of vocal fold vibration was expressed as a fraction of the cuneiform cartilage size in order to reduce potential errors caused by movement of the laryngoscope and larynx during videolaryngostroboscopy.

Other studies have employed a similar methodology, having used the distance between the anterior commissure and the vocal process as a reference (Omori et al., 1996; Tsuji et al., 2003). We were unable to use that measurement in our study because we noted a change in glottal configuration during tongue trills, as reported in a study by Bueno (2006), who observed anteroposterior constriction of the laryngeal vestibule, without medialization or vibration of the vestibular vocal fold, during tongue trills.

The proximity of the distal end of the laryngoscope to the larynx can generate barrel distortion of linearity (Lee, 1980). In that type of distortion, the proportion of the measurements in the center of the image (represented in the present study as the vibration amplitude measurement) is smaller than is that of those in the periphery (represented as the cuneiform cartilage measurement) for the same millimetric measurement (Lee, 1980). Of the 220 images analyzed in the present study, $163(74 \%)$ were indicative that the laryngoscope was far enough from the larynx to avoid that type of distortion. Of the images that were indicative that the laryngoscope was closer to the larynx and that could therefore show distortion, 21\% (46/220) were obtained during the trill exercises and 5\% (11/220) were obtained during the sustained vowel phonation task.

We believe that those distortions were not enough to interfere with the present study, given that the amplitude of vocal fold vibration was greater during the trill exercises than during 
the sustained vowel phonation task, even when the image was indicative that the laryngoscope was close to the larynx.

There were significant differences among phonation of the sustained vowel $/ \varepsilon /$, lip trills, and tongue trills in terms of the maximum amplitude of vocal fold vibration at low and high intensities ( $p=0.002$ and $p=0.001$, respectively). The Wilcoxon signed rank test revealed significant differences between lip trills and phonation of the sustained vowel $/ \varepsilon /$, as well as between tongue trills and phonation of the sustained vowel $/ \varepsilon /$.

For the performance of lip trills and tongue trills, pulmonary airflow has to increase in order to maintain vocal fold vibration and lip or tongue vibration (Titze, 2009; Warren et al., 1992; Titze 1988, McGowan, 1992). The increase in airflow can lead to an increase in subglottic pressure, which can in turn lead to an increase in the amplitude of vocal fold vibration (Garrel et al., 2008; Titze, 2009).

In the present study, during the measurement of amplitude, it was difficult to synchronize the flashes of light from the stroboscope with the vocal fold vibration during lip and tongue trills in some cases. We had no such difficulty when we measured the amplitude of vocal fold vibration during phonation of the sustained vowel $/ \varepsilon /$. Therefore, during lip trills and tongue trills, we photographed only those moments of the cycles at which the maximum opening was visible.

Increased airflow can also lead to aperiodicity of vocal fold vibration (Jiang, 2001; Tao, 2007) and can destabilize the flashes of light from the stroboscope (Sercarz et al., 1992). In previous studies, we found that the standard deviation of the intraindividual closed quotient was highest during lip trills and tongue trills, the values being higher at high intensities, which is due to increased airflow (Russell et al., 1998; Alku, 2006). Those results might indicate differences in the periodicity of the EGG waveform during trill exercises and corroborate the abovementioned hypothesis.

Bueno (2006) observed pronounced movement of the laryngeal framework during tongue trills; such movement can make it difficult for the contact microphone to pick up the fundamental frequency. Because the cycles observed under the stroboscopic light are not the actual vocal fold vibration cycles, which require regularity in order to generate reliable images (Sercarz et al., 1992; Patel et al., 2008), a study involving a high-speed camera would be needed in order to have a better view of vocal fold vibration during tongue and lip trills. To that end, a device that is compatible with the laryngoscope is needed. Studies involving a qualitative analysis of both the periodicity of the EGG waveform and the amplitude of the EGG signal could also be useful in the investigation of the mechanism of vocal fold vibration during trill exercises.

The standard deviation of the closed quotient is a numerical representation of the variation in the closed quotient in the EGG waveform. Because the standard deviation of the closed quotient was higher during the trill exercises (at high or low intensity) in the present study, we can affirm that the variation in the closed quotient is greater during trill exercises than during phonation of the sustained vowel $/ \varepsilon /$.

For tongue trills and lip trills to occur, the anterior part of the vocal tract has to be occluded by the tongue or the lips. Intraoral pressure increases and becomes greater than the force 
that maintains the anterior part of the vocal tract closed; therefore, the anterior part of the vocal tract opens and is "sucked out" by the speed of the airflow (McGowan, 1992), closing the vibration cycle, as occurs in the vocal fold vibration model. Therefore, supraglottic pressure and, consequently, vocal tract impedance oscillate.

The theory of source-filter interaction (Titze, 2008; Titze et al., 2008) states that the acoustic pressure in the vocal tract changes the phonation threshold pressure and interferes with vocal fold vibration. The theory of source-filter interaction states that, when the vibratory motion of the point of articulation makes the acoustic pressure in the vocal tract oscillate, the phonation threshold pressure also oscillates, which might explain why the standard deviation of the closed quotient was higher during trill exercises in the present study.

According to Titze (2006), the objective of voice training is to promote the interaction between the source and the filter and therefore increase vocal intensity, efficiency, and economy (Titze, 2006). According to the author, lip trills and tongue trills are among the semi-occluded vocal tract exercises. Because semi-occluded vocal tract exercises promote a mechanical interaction between the source and the filter (Titze, 2008; Titze et al., 2008) they change vocal fold impedance and therefore inhibit vocal fold vibration (Story et al., 2000).

Vocal tract pressure during voiced fricatives must be constant, which distinguishes voiced fricatives from the exercises analyzed in the present study. New clinical studies comparing the two types of exercises should be conducted.

In our studies, the mean closed quotient was highest during lip trills at high intensity, which distinguished lip trills from tongue trills and phonation of the sustained vowel $/ \varepsilon /$. According to the literature (Story et al., 2000; Titze, 2006), a more anterior obstruction translates to greater vocal tract impedance. Although lip trills are slightly more anterior than are tongue trills, the influence of supraglottic and subglottic pressure on vocal fold vibration is not linear (Zhang, 2009; Titze, 2008; Hatzikirou et al., 2006, Titze, 2008), meaning that the airflow changes caused by increased intensity can lead to different vocal fold vibration proportions and differentiate between high- and low-intensity vibrations (Tao et al., 2007; Becker et al., 2009).

Gaskill and Erikson (2008) found systematic differences between the closed quotient of lip trills and that of phonation of the sustained vowel $/ \varepsilon /$; as we did in the present study, the authors argued that those differences might be due to the interaction between the source and the filter. In addition, the authors found differences between trained and untrained individuals in terms of the results obtained; the differences were attributed to the fact that trained individuals have better control over glottic closure.

Some authors have reported that the sound produced by the larynx is not linear (Jing et all, 2001) and depends on numerous factors. Therefore, any difference in biomechanics, structure (such as tissue geometry, density, and viscosity), airflow control, or vocal tract control can cause differences in vocal fold vibration. Trained individuals have more control over those factors, and this can actually result in differences between trained and untrained individuals, as well as between trained individuals and patients with morphological changes in the vocal folds, in terms of vocal fold vibration during a given phonation task. 
Because patients with vocal fold pathologies present with structural changes (and, consequently, biomechanical changes), trill exercises probably produce different effects in those individuals than in individuals without morphological changes. According to the literature, bulging caused by vocal fold pathologies interfere with glottal flow resistance, glottal width, glottal area, and mean glottal volume velocity (Alipour \& Scherer, 2000).

Studies involving vocal exercises (including tongue and lip trills) in various settings should be conducted in order to provide a deeper understanding of the physiology of vocal exercises in each of those situations and therefore assist speech-language pathologists in prescribing the exercises. The present study can support some of the theories that underlie the use of trill exercises in the clinical practice of speech-language pathology, as well as in the voice training of professional voice users. According to McGowan (1996), the variations that occur in the pharynx during lip trills can increase the force of mucosal vibration during the wavelike motion of the vocal folds.

The greater amplitude of mucosal vibration and the higher standard deviation of the closed quotient during trill exercises reflect changes in the wavelike motion of the vocal folds; this can explain, at least in part, the improvement in voice quality (Rodrigues, 1995) after the use of those exercises, as well as warranting the use of trill exercises in patients with vocal fold pathologies, such as nodules (Bueno, 2006), edema (Pinho e Pontes, 2008), and sulci. The probable need for airflow control and the source-filter interaction caused by the articulatory oscillation warrant the use of trill exercises during the training of professional voice users, as recommended by Aydos and Hanayama (2004) and Nix (1999).

However, further studies are needed in order to provide a deeper understanding of the effects of trill exercises on the vocal fold mucosa. To that end, a more in-depth analysis of the EGG waveform and, if possible, videolaryngostroboscopy with a high-speed camera are warranted.

For a better understanding of the effect of trill exercises and their indications, studies analyzing the blood flow in the region and the mechanics of laryngeal and vocal tract muscles during the exercises are needed.

\section{Conclusion}

On the basis of our results, we can conclude that, in professional singers, the maximum amplitude of vocal fold vibration is greater during lip and tongue trills than during natural phonation. In addition, we observed considerable variation in the closed quotient during the exercises employed. Lip trills differed from tongue trills only at higher intensities and in terms of the closed quotient.

\section{References}

Alku P.; Airas M.; Bjorkner E. \& Sundberg J. (2006) An amplitude quotient based method to analyze changes in the shape of the glottal pulse in the regulation of vocal intensity. J Acoust Soc Am. 120(2):1052-62. ISSN 0001-4966 
Aydos B \& Hanayama M (2004). Técnicas de aquecimento vocal utilizadas por professores de teatro. Rev CEFAC: 6(1): 83-8. ISSN 1516-1846.

Becker S; Kniesburges S; Muller S; Delgado A; Link G; Kaltenbacher M, et al. (2009). Flowstructure-acoustic interaction in a human voice model. J Acoust Soc Am;125(3):135161. ISSN 0001-4966

Behlau M \& Pontes P (1995). Avaliação e tratamento das disfonias: Editora Lovise. Rio de Janeiro. ISBN 8585274263

Bueno TC (2006). Técnica de vibração sonorizada de língua: aspectos do aprendizado, dos efeitos acústicos e das imagens do trato vocal e da face [mestrado]. São Paulo PUC-São Paulo; 2006.

Casper J; Colton R; Woo P \& Brewer D. (1994) Physiological characteristics of selected voice therapy techniques: a preliminary research note. British Voice Assoc;1:131-41. 3873295.

Cordeiro GF; Montagnoli AN; Nemr NK; Menezes MHM \& Tsuji DH. Comparative analysis of the closed quotient for lip and tongue trill in relation to the sustained vowel $/ \varepsilon /$. J Voice. In Prelo. ISSN 0892-1997.

Garrel R; Scherer R; Nicollas R; Giovanni A \& Ouaknine M (2008). Using the relaxation oscillations principle for simple phonation modeling. J Voice. 22:385-98. ISSN 08921997

Gaskill CS \& Erickson ML. (2008) The effect of a voiced lip trill on estimated glottal closed quotient. J Voice.22(6):634-43. ISSN 0892-1997

Gregio FN. (2006) Configuração do trato vocal supraglótico na produção das vogais do Português: dados de imagem de ressonância magnética [Mestrado]. São Paulo: PUC-SP; 2006.

Hatzikirou H; Fitch WT \& Herzel H. (2006) Voice Instabilities due to Source-Tract Interactions. Acta acustica united with acustica 92468 - 75.

Jiang JJ, Zhang Y, Stern J. (2001) Modeling of chaotic vibrations in symmetric vocal folds. J Acoust Soc Am. 110(4):2120-8. ISSN 0001-4966

Lee KH. (1980) Quantitative assessment of linearity of scintillation cameras. Radiology. 136(3):790-2. ISSN 1527-1315.

Linklater K (1976). Freeing the natural voice. New York: Drama Book. ISBN 97800896760714.

Maniecka-Aleksandrowicz B; Domeracka-Kolodziej A; Rozak-Komorowska A \& SzeptyckaAdamus A. (2006)Management and therapy in functional aphonia: analysis of 500 cases. Otolaryngol Pol. 2006;60(2):191-7. ISSN 0030-6657

McGowan RS. (1992) Tongue-tip trills and vocal-tract wall compliance. J Acoust Soc Am. 91(5):2903-10. ISSN 0001-4966.

Menezes MH; Duprat AC \& Costa HO (2005). Vocal and laryngeal effects of voiced tongue vibration technique according to performance time. J Voice 22(5): 565-80. ISSN 08921997.

Nix J. (1999) Lip Trills and Raspberries: "High Spit Factor" Alternatives to the Nasal Continuant Consoants Journal of Singing.55(3):15-9. ISSN 1086-7732. 
Omori K; Kacker A; Slavit DH \& Blaugrund SM (1996). Quantitative videostroboscopic measurement of glottal gap and vocal function: an analysis of thyroplasty type I. Ann Otol Rhinol Laryngol. 105(4):280-5. ISSN 0196-0709.

Patel R; Dailey S \& Bless D. (2008) Comparison of high-speed digital imaging with stroboscopy for laryngeal imaging of glottal disorders. Ann Otol Rhinol Laryngol. 117(6):413-24. . ISSN 0196-0709.

Pinho SM; Tsuji DH \& Bohadana SC (2006) Tratamento fonoaudiológico das disfonias dirigidos à fonocirurgia. In: Pinho SM, Tsuji DH, S.C B, editors. Fundamentos em Laringologia e Voz. Rio de Janeiro: Revinter; p. 69 - 84. ISBN 9788573099879.

Rodrigues MRC. (2001) Estudo do exercício de vibração sonorizada de língua nas laringectomias frontolaterais. [Mestrado]. São Paulo: PUC-São Paulo.

Rodrigues S. (1995) Análise múltipla do efeito da técnica de vibração sonorizada de língua em indivíduos adultos sem queixa vocal. [Mestrado]. São Paulo: Universidade Federal de São Paulo.

Russell BA; Cerny FJ \& Stathopoulos ET. (1998) Effects of varied vocal intensity on ventilation and energy expenditure in women and men. J Speech Lang Hear Res. 41(2):239-48. ISSN 1558-9192.

Sataloff RT (1991). Professional Voice: The science and art of clinical care. New York: Raven Press ISBN 1565937287.

Schneider SL \& Sataloff RT (2007). Voice therapy for the professional voice. Otolaringol Clin $N$ Am 40 1133-49. ISSN 030-6665.

Schwarz K \& Cielo CA (2009) Vocal and larybgeal modifications produced by the sonorous tongue vibration techinique. Pro Fono 21(2): 161:6. ISSN 0104-5687

Sercarz JA, Berke GS, Gerratt BR, Kreiman J, Ming Y, Natividad M (1992). Synchronizing videostroboscopic images of human laryngeal vibration with physiological signals. Am J Otolaryngol. 13(1):40-4. ISSN 0196-0709.

Speyer R. (2008) Effects of voice therapy: a systematic review. J Voice. 22:565-80. ISSN 08921997

Story BH; Laukkanen AM \& Titze IR. (2000) Acoustic impedance of an artificially lengthened and constricted vocal tract. J Voice. 14(4):455-69. ISSN 0892-1997.

Story BH; Titze IR \& Hoffman EA. (1998) Vocal tract area functions for an adult female speaker based on volumetric imaging. J Acoust Soc Am 104(1):471-87. ISSN 00014966.

Tao C; Zhang Y; Hottinger DG \& Jiang JJ. (2007) Asymmetric airflow and vibration induced by the Coanda effect in a symmetric model of the vocal folds. J Acoust Soc Am. 122(4):2270-8. ISSN 0001-4966.

Titze IR. (2006) Voice training and therapy with a semi-occluded vocal tract: rationale and scientific underpinnings. J Speech Lang Hear Res. 49(2):448-59. ISSN 1558-9192.

Titze IR. (2009) Phonation threshold pressure measurement with a semi-occluded vocal tract. J Speech Lang Hear Res. 52(4):1062-72. ISSN 1558-9192

Tsuji DH; de Almeida ER; Sennes LU; Butugan O \& Pinho SM. (2003) Comparison between thyroplasty type I and arytenoid rotation: a study of vocal fold vibration using excised human larynges. J Voice. 17(4):596-604. ISSN 0892-1997. 
Vieira MN; McInnes FR \& Jack MA. (1997) Comparative assessment of electroglottographic and acoustic measures of jitter in pathological voices. J Speech Lang Hear Res. 40(1):170-82. ISSN 1558-9192

Warren DW; Rochet AP; Dalston RM \& Mayo R. (1992) Controlling changes in vocal tract resistance. J Acoust Soc Am. 91(5):2947-53. ISSN 0001-4966

Woo P; Casper J; Colton R \& Brewer D. (1994) Diagnosis and treatment of persistent dysphonia after laryngeal surgery: a retrospective analysis of 62 patients. Laryngoscope. 104(9):1084-91. ISSN 1531-4995

Zhang Z. (2009) Characteristics of phonation onset in a two-layer vocal fold model. J Acoust Soc Am. 125(2):1091-102. ISSN 0001-4966 


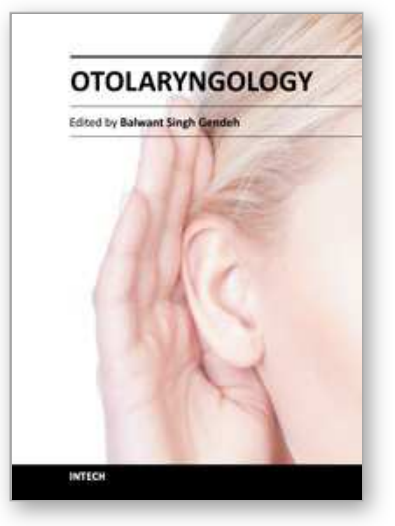

\author{
Otolaryngology \\ Edited by Prof. Balwant Singh Gendeh
}

ISBN 978-953-51-0624-1

Hard cover, 198 pages

Publisher InTech

Published online 23, May, 2012

Published in print edition May, 2012

This book emphasizes on different aspects of otolaryngology - the medical sciences of diagnosis and treatment of ENT disorders. "Otolaryngology" is divided into various clinical sub-specialities, namely otology, rhinology, laryngology, and head and neck. This book incorporates new developments, as well as future perspectives in otolaryngology. I would like to dedicate this book to those of you who will pick up the torch and by continued research, close clinical observation and the highest quality of clinical care, as well as by publication and selfless teaching, further advance knowledge in otolaryngology from this point forward. It is intended to be a guide to other books to follow. Otolaryngologists, researches, specialists, trainees, and general practitioners with interest in otolaryngology will find this book interesting and useful.

\title{
How to reference
}

In order to correctly reference this scholarly work, feel free to copy and paste the following:

Gislaine Ferro Cordeiro, Arlindo Neto Montagnoli and Domingos Hiroshi Tsuji (2012). Comparison Among Phonation of the Sustained Vowel/ع/, Lip Trills, and Tongue Trills: The Amplitude of Vocal Fold Vibration and the Closed Quotient, Otolaryngology, Prof. Balwant Singh Gendeh (Ed.), ISBN: 978-953-51-0624-1, InTech, Available from: http://www.intechopen.com/books/otolaryngology/comparative-analysis-of-the-amplitude-ofvibraition-of-the-vocal-folds-and-the-coefficient-of-contac

\section{INTECH}

open science | open minds

\author{
InTech Europe \\ University Campus STeP Ri \\ Slavka Krautzeka 83/A \\ 51000 Rijeka, Croatia \\ Phone: +385 (51) 770447 \\ Fax: +385 (51) 686166 \\ www.intechopen.com
}

\author{
InTech China \\ Unit 405, Office Block, Hotel Equatorial Shanghai \\ No.65, Yan An Road (West), Shanghai, 200040, China \\ 中国上海市延安西路65号上海国际贵都大饭店办公楼 405 单元 \\ Phone: +86-21-62489820 \\ Fax: +86-21-62489821
}


(C) 2012 The Author(s). Licensee IntechOpen. This is an open access article distributed under the terms of the Creative Commons Attribution 3.0 License, which permits unrestricted use, distribution, and reproduction in any medium, provided the original work is properly cited. 